\title{
Light sheet fluorescence microscope for microfluidic chip
}

\author{
Caroline Bissardon ${ }^{1}$, Xavier Mermet $^{1}$, Sophie Morales ${ }^{1}$, Frédéric Bottausci ${ }^{1}$, Marie Carriere ${ }^{2}$, Florence \\ Rivera $^{1}$, and Pierre Blandin ${ }^{1, *}$ \\ ${ }^{1}$ Univ. Grenoble Alpes, CEA, LETI, DTBS, F-38000 Grenoble, France \\ ${ }^{2}$ Univ. Grenoble-Alpes, CEA, CNRS , IRIG, SyMMES, F-38000 Grenoble, France
}

\begin{abstract}
We present a light sheet fluorescence microscope dedicated to image "Organ-on-chip"-like biostructures in microfluidic chip. Based on a simple design, the setup is built around the chip and its environment to allow 3D imaging inside the chip in a microfluidic laboratory. The experimental setup, its optical characterization and first volumetric images are reported.
\end{abstract}

\section{Introduction}

Nowadays, the organ-on-chip technology presents a large interest for biomedical researches. Important issues, such as the vascularization of organoids or tissues, remain and must be solved to enhance the potential of this application technique.

In the present study, the optical system has been developed to response to vascularization problems encountered in Organ-on-chip imaging. The visualization and understanding of the capillarization process occurring within the organoid-like samples is crucial to characterize it. To follow up the vascularization process, a microfluidic chip associated with an adequate microfluidic injection system have been developed at the CEA LETI.

Obtaining information of the central core of the sample is not satisfying with classical fluorescence microscopy techniques such as inverted fluorescence microscope, spinning-disk microscopy. Light Sheet Fluorescence Microscopy (LSFM) seems to be a promising solution to analyze the inner parts of samples. The main interest of this microscopy is to allow a 3D morphological and functional characterization of organoids and other tissues cultured in a microfluidic chamber. The samples will not be transformed or clarified before analyses in order to be physico-chemically and photo-preserved.

LSFM is an optical imaging technique that has been increasingly used in biological applications ranging from molecular biology to whole mount tissue analysis. This technique presents a lot of advantages in microdevice field $^{1-5}$. The basic idea of this technique is described in the reviews ${ }^{6-8}$. This confinement of laser light confers optical sectioning capability to the microscope. The main advantages of this technique are the high-speed volumetric acquisition rate and the low photo-toxicity ${ }^{6-8}$. These two particularities are essential for rapid 3D but also for 4D imaging.

\section{Optical microscope system}

\subsection{Positioning}

To limit the disturbance in the sample development and avoid any microfluidic injection problem, the image acquisition need to be done on a horizontal microfluidic chip. Inspired by the inverted selective plane illumination microscopy developed by $\mathrm{Wu}$ et al. $2011^{9}$, we conceived a new microscope for specific "Organ-on-chip" application. Long distance objective is required to be able to collect the fluorescence coming from the microfluidic disposal system and having enough space to insert, position and move it. The detection and excitation paths are placed at 90 degrees from each other on a vertical breadboard in order to observe horizontally the sample positioned at 45 degrees from both optical arms. The light sheet (LS) is controlled with a cylindrical lens in order to form a fine and homogeneous LS on the field of view of the camera. 3D imaging is then possible thanks to the translation of this LS at different depth of the sample. Contrary to other studies focusing on super-resolution or large field of view, the main purpose of this instrument is to obtain the best compromise between large field of view and resolution that can be achieved to morphologically analyze samples.

\subsection{Experiment set-up}

The beam from a $488 \mathrm{~nm}$ Ar laser is enlarged with an afocal system. The expanded Gaussian laser beam is shaped into a light sheet with a cylindrical lens. This laser plane illuminates the sample contained in the microfluidic chip with an angle of 45 degrees, as shown in Fig. 1. The microfluidic chip is mounted on translation stages in order to be able to scan the whole sample. The fluorescence from the sample plane illuminated with the light sheet is collected by a long working distance $20 x$ objective,

\footnotetext{
* Corresponding author: pierre.blandin@cea.fr
} 
associated with a long-pass filter and a tube lens. The wide-field image from this orthogonal viewing direction is acquired on a sCMOS camera.

Fig. 1. Scheme of the experimental set-up of the light-sheet fluorescent microscope for microfluidic analysis on chip realized with Solidworks. Disposal centered on the sample holder system with an inset presenting the support of the microfluidic chip and the microfluidic injection system location.

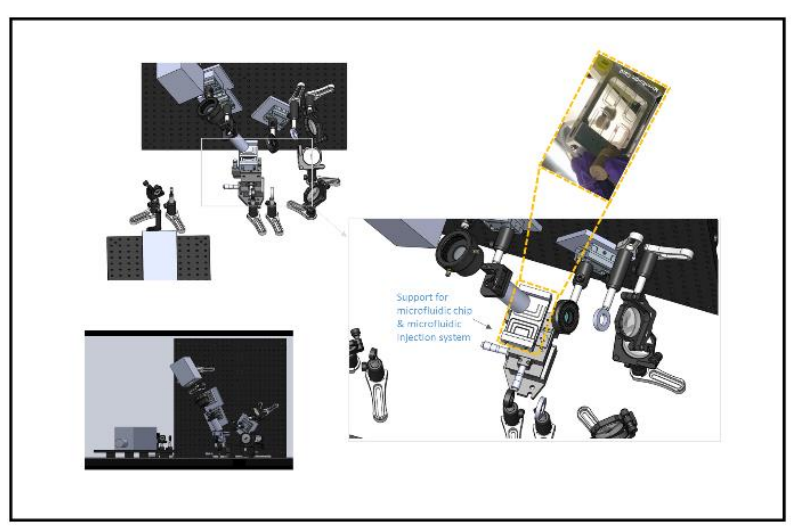

\subsection{Characterisation of the optical system}

The system was classically optically characterized using green-yellow fluorescent microspheres with a size lower than the expected resolution. These beads were disposed on a classical microscope slide to determine the spatial resolution of this microscope system. We imaged a xy plane parallel to the camera which corresponds to a plane at 45 degrees from the microfluidic chip. Fig. 2 shows the xy plane of the selected ROI and the corresponding intensity profiles of the PSF along $\mathrm{x}$ - and $\mathrm{y}$-directions. The fitted Gaussian curve gives a value of $\sim 3 \pm 0.3 \mu \mathrm{m}$ for the lateral resolution measured at the FWHM. We also investigated the resolution that can be achieved within the microfluidic chamber with these same fluorescent microbeads. We proceeded with different buffer solutions in order to estimate their potential influences. Furthermore, we imaged in 3D diverse volumetric samples (pollen, distribution of several substances, and characterization of the chamber structures...). 3D rending can then be obtained with data treatments and analysis. Further experiments on biological samples are currently on-going and could be very soon presented.

\section{Conclusion}

The development of this LSFM leads to an innovative microscope dedicated to imaging in microfluidic chip. The system being well characterized, we are currently implementing the next step, which consists in realizing a real time acquisition on a $3 \mathrm{D}$ volume of a continuously perfused biological sample. This simple set-up presented will probably be optimized for the biological sample of interest that will be studied. The set-up must stay easy to handle with a minimal data analysis.
Fig. 2. xy plane of the selected ROI and the corresponding intensity profiles of the PSF along $\mathrm{x}$-direction and $\mathrm{y}$ direction with the fitted Gaussian curve associated. White scale bar: $25 \mathrm{~m}$

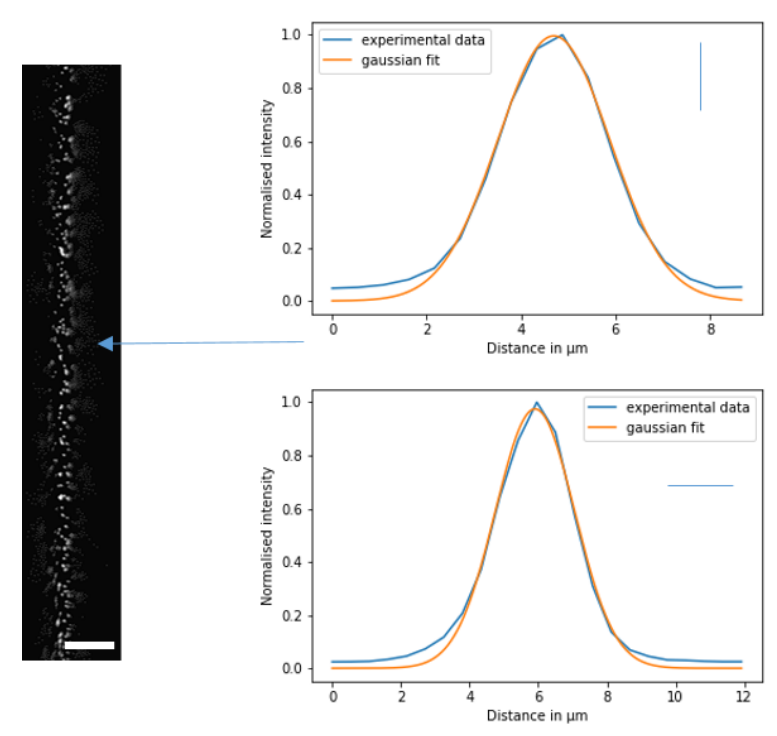

We acknowledge the financial support of the Cross-Disciplinary Program on Instrumentation and Detection of CEA, the French Alternative Energies and Atomic Energy Commission.

\section{References}

1. I. Albert-Smet, A. Marcos-Vidal, JJ. Vaquero, M. Desco, A. Muñoz-Barrutia, J. Ripoll. Front Neuroanat.13:1 (2019)

2. E. De Santis, E. Shardlow, F. Stellato, et al. Condens Matter.;4(1):13 (2019)

3. R. Galland, G. Grenci, A. Aravind, V. Viasnoff, V. Studer, JB. Sibarita. 3 Nat Methods.;12:641 (2015)

4. A-K. Gustavsson, PN. Petrov, WE. Moerner.. Opt Express.;26(10):13122-13147. (2018)

5. JW. Krieger, AP. Singh, CS. Garbe, T. Wohland, J. Langowski. Opt Express.22(3):2358-2375 (2014)

6. J. Huisken,J. Swoger, F. Del Bene, J. Wittbrodt, EHK Stelzer. Science (80-).;305(5686):1007(2004)

7. OE. Olarte, J. Andilla, EJ. Gualda,P. Loza-Alvarez P. Adv Opt Photonics.10(1):111 (2018)

8. LA. Royer, WC . Lemon, RK. Chhetri, et al. Nat Biotechnol.34(12):1267-1278 (2016)

9. Y. Wu, A. Ghitani, R. Christensen, et al. Proc Natl Acad Sci US A.;108(43):17708-17713 (2011) 